FERMILAB-TM-1995

\title{
D $\varnothing$ Luminosity Monitor Constant for the 1994-1996 Tevatron Run
}

\author{
J. Bantly, J. Krane, D. Owen, R. Partridge and L. Paterno
}

Fermi National Accelerator Laboratory

P.O. Box 500, Batavia, Illinois 60510

June 1997 


\section{Disclaimer}

This report was prepared as an account of work sponsored by an agency of the United States Government. Neither the United States Government nor any agency thereof, nor any of their employees, makes any warranty, expressed or implied, or assumes any legal liability or responsibility for the accuracy, completeness, or usefulness of any information, apparatus, product, or process disclosed, or represents that its use would not infringe privately owned rights. Reference herein to any specific commercial product, process, or service by trade name, trademark, manufacturer, or otherwise, does not necessarily constitute or imply its endorsement, recommendation, or favoring by the United States Government or any agency thereof. The views and opinions of authors expressed herein do not necessarily state or reflect those of the United States Government or any agency thereof.

\section{Distribution}

Approved for public release; further dissemination unlimited. 
Fermilab-TM-1995

DØ Note 3199

Feb. 1, 1997

\title{
DØ Luminosity Monitor Constant for the 1994-1996 Tevatron Run
}

\author{
J. Bantly, J. Krane, D. Owen, R. Partridge, L. Paterno
}

\begin{abstract}
The D $\emptyset$ experiment calculates its luminosity using the visible cross section (luminosity monitor constant) $\sigma_{\mathrm{L} \emptyset}$ for its Level $\emptyset$ trigger based on the world average $\bar{p} p$ inelastic cross sections at $\sqrt{s}=1.8 \mathrm{TeV}$. For the 1992-1993 Tevatron Run (1A), the luminosity monitor constant was determined to be $\sigma_{\mathrm{L} \emptyset}=46.7 \pm 2.5 \mathrm{mb}$. For the 1994-1996 Tevatron Run $(1 \mathrm{~B} / 1 \mathrm{C})$, the luminosity monitor constant changed slightly with an upgrade to the electronics in the Level $\emptyset$ trigger. The luminosity monitor constant becomes on average $\sigma_{\mathrm{L} \emptyset}=44.53 \pm 2.37 \mathrm{mb}$ for Run 1B/1C. In addition, small corrections for halo and multiple single diffraction are included in integrated luminosity determinations.
\end{abstract}

\section{Introduction}

The $\mathrm{D} \emptyset$ luminosity monitor constant, $\sigma_{\mathrm{L} \emptyset}$, is a measure of the visible inelastic cross section of the $\mathrm{D} \emptyset$ detector [1]. A previous determination of $\sigma_{\mathrm{L} \emptyset}$ was made for the 1992-1993 Tevatron run and details are found elsewhere [2]. Due to a change in the electronics for the LØ trigger [3] at the beginning of the 1994-1996 Tevatron run, the hardware efficiency for the L $\varnothing$ trigger has been redetermined. The acceptances for the L $\emptyset$ trigger and the world average cross sections for total, elastic, and single diffractive collisions [4] are unchanged as summarized in Appendix A.

The calculation of $\sigma_{\mathrm{L} \emptyset}$ is given by Equation 1 .

$$
\sigma_{\mathrm{L} \emptyset}=\epsilon_{\mathrm{L} \emptyset}\left(\epsilon_{\mathrm{SD}} \sigma_{\mathrm{SD}}+\epsilon_{\mathrm{DD}} \sigma_{\mathrm{DD}}+\epsilon_{\mathrm{HC}} \sigma_{\mathrm{HC}}\right)
$$


where $\epsilon_{\mathrm{SD}}=$ single diffractive acceptance in the $\mathrm{L} \emptyset$ array, $\sigma_{\mathrm{SD}}=$ world average single diffractive cross section and $\epsilon_{\mathrm{DD}}, \sigma_{\mathrm{DD}}, \epsilon_{\mathrm{HC}}, \sigma_{\mathrm{HC}}$ are similar terms for double diffractive and hard core inelastic collisions. These acceptances are determined by treating the $L \emptyset$ hardware as $100 \%$ efficient. A determination of the $L \emptyset$ hardware efficiency factor $\epsilon_{\mathrm{L} \emptyset}$ is made using $\mathrm{D} \emptyset$ zerobias data. This paper addresses the change in $\sigma_{\mathrm{L} \varnothing}$ due to a change in the L $\emptyset$ hardware efficiency factor $\epsilon_{\mathrm{L} \varnothing}$ and the addition of two corrections to the $\sigma_{\mathrm{L} \varnothing}$ calculation, the halo $\left(f_{\text {halo }}\right)$ and multiple single diffraction $\left(f_{\mathrm{MSD}}\right)$ correction factors.

During the 1994-1996 run, several unbiased data samples were collected by the $\mathrm{D} \emptyset$ detector over a wide range of luminosities. These samples were used to determine the $L \emptyset$ efficiency over the full luminosity range. In addition, due to peak luminosities of over $20.0 \times 10^{30} \mathrm{~cm}^{-2} \mathrm{~s}^{-1}$, correction factors for beam halo and multiple single diffractive effects have been included.

\section{The Level $\varnothing$ Trigger}

The $L \emptyset$ hardware trigger is a fast triggering system for the $D \emptyset$ detector. Details of the $L \emptyset$ detector and its electronics are available in the $L \emptyset$ trigger reference [3]. The $L \emptyset$ trigger indicates which beam crossings, occurring every $3.5 \mu \mathrm{s}$, contain non-diffractive inelastic collisions and monitors the instantaneous luminosity. The $L \emptyset$ detector is comprised of two hodoscope scintillator arrays located on the inside faces of the $D \emptyset$ detector's two end calorimeters, $140 \mathrm{~cm}$ from the center of the detector and perpendicular to the beam direction. Each array partially covers a region in pseudorapidity of $1.9<|\eta|<4.3$, with nearly complete coverage over $2.2<|\eta|<3.9$. The north array covers the negative $\eta$ region and the south array covers the positive $\eta$ region. Analog sums are formed using the signals from the photomultipier tubes connected to the $20 \mathrm{~L} \emptyset$ counters closest to the beampipe for each of the north and south L $\emptyset$ arrays. These two analog sums pass through constant fraction discriminators and feed into the FASTZ electronics module. The module uses the difference in arrival times of the two sums to calculate a collision vertex position along the beam direction $(z)$. "Good" FASTZ triggers have a $|z|<96 \mathrm{~cm}$, where $z=0$ is located at the center of the $\mathrm{D} \emptyset$ detector. The FASTZ trigger counts beam crossings with inelastic collisions, thus determining the luminosity at $D \emptyset$. In addition, the $L \emptyset$ 
trigger provides measurements of beam halo and can be used to calculate beam crossing angles [5]. It also serves as a minimum bias trigger for physics analyses.

The discriminator outputs of the two analog sum signals also feed into Flash ADCs from which the individual arrival times can be obtained with an accuracy of approximately 1 nsec. A comparison between the FASTZ module and a calculation made using the FADC times is available for diagnostic purposes. The FADC times can also determine if there were hits in both $\mathrm{L} \emptyset$ counters in time with the beam crossing; the FASTZ module only provides a time difference and not two absolute times. Additional processing of the LØ arrays' individual channels provides a measurement of the interaction $z$-position for use in later trigger decisions (SLOWZ) and flags the presence of multiple interactions.

The electronics pathways for the PMT signals before the analog sums were altered for the 1994-1996 Tevatron run to improve the FASTZ $z$ resolution. This improvement in the $z$ resolution by a factor of 2.5 also decreased the efficiency of the FASTZ Level $\varnothing$ trigger. This note contains the results of the study to calculate $\epsilon_{\mathrm{L} \emptyset}$ for the 1994-1996 Tevatron run.

\section{Instantaneous Luminosity based on L $\varnothing$ Scaler Rates}

Consider the average number of interactions per beam crossing $\bar{n}$,

$$
\bar{n}=\mathcal{L} \tau \sigma
$$

or, rewritten

$$
\mathcal{L}=\bar{n} / \tau \sigma
$$

where $\tau=3.5 \mu \mathrm{s}$ is the time interval between crossings, $\sigma$ is the visible cross section and $\mathcal{L}$ is the instantaneous luminosity. The probability of $n$ interactions in one crossing, $\mathcal{P}_{n}$, follows Poisson statistics and is given by,

$$
\mathcal{P}_{n}=\frac{\mu^{n}}{n !} e^{-\mu}
$$

where $\mu$ is just $\bar{n}$, the mean number of interactions per beam crossing. 
At D $\emptyset$ we measure $R_{\mathrm{L} \emptyset}$, the rate of good FASTZ L $\emptyset$ triggers. $R_{\mathrm{L} \emptyset}$ does not count individual interactions in a beam crossing but counts only once for each crossing with one or more interactions. Using the time between crossings, $\tau$, the fraction of crossings with one or more interactions is given by

$$
R_{\mathrm{L} \emptyset} \tau=1-\mathcal{P}_{0}
$$

where $\mathcal{P}_{0}=e^{-\bar{n}}$ is the fraction of crossings with no interactions. Substituting into Eqn. 3,

$$
\mathcal{L}=\frac{-\ln \left(1-R_{\mathrm{L} \emptyset} \tau\right)}{\sigma_{\mathrm{L} \emptyset} \tau}
$$

where $\sigma_{\mathrm{L} \emptyset}$ is the luminosity monitor constant at $\mathrm{D} \emptyset$. This calculation takes into account the multiple interactions that can occur within each beam crossing. Because the bunches in the machine were not all identical, the luminosity was measured separately for each of the six bunches in the Tevatron and the results summed. The calculation for each bunch is identical except with a $\tau$ value six times greater.

\section{The L $\varnothing$ Trigger Efficiency}

The L $\varnothing$ trigger hardware efficiency factor, $\epsilon_{\mathrm{L} \emptyset}$, is defined as the efficiency with which the L $\emptyset$ trigger fires on a single, inelastic interaction. This efficiency should then be luminosity independent. The L $\varnothing$ trigger efficiency factor was determined by using samples of $D \emptyset$ data collected while triggering on random beam crossings (zero bias). These unbiased data samples are collected by triggering the $\mathrm{D} \emptyset$ data acquisition system [1] solely on the crossing time of the $\bar{p} p$ beams at the $\mathrm{D} \emptyset$ collision region rather than on the detected presence of an interaction.

We measure the $\mathrm{L} \emptyset$ pedestals using zero bias data events with no $\mathrm{L} \emptyset$ hits coincident with a beam crossing. The distributions of charge from the analog sums are shown in Figures 1(a) and 2(a) revealing the locations of the pedestal peaks for the two arrays. The double peaked nature of the pedestal is a result of electronics noise. A cross-check on pedestal shape was provided by looking at the charge sum when no central tracking vertex was found. Compare Figures 1 and 2. Although the pedestal selection used for this study involves Level $\emptyset$ itself, a comparison of the histograms indicates 

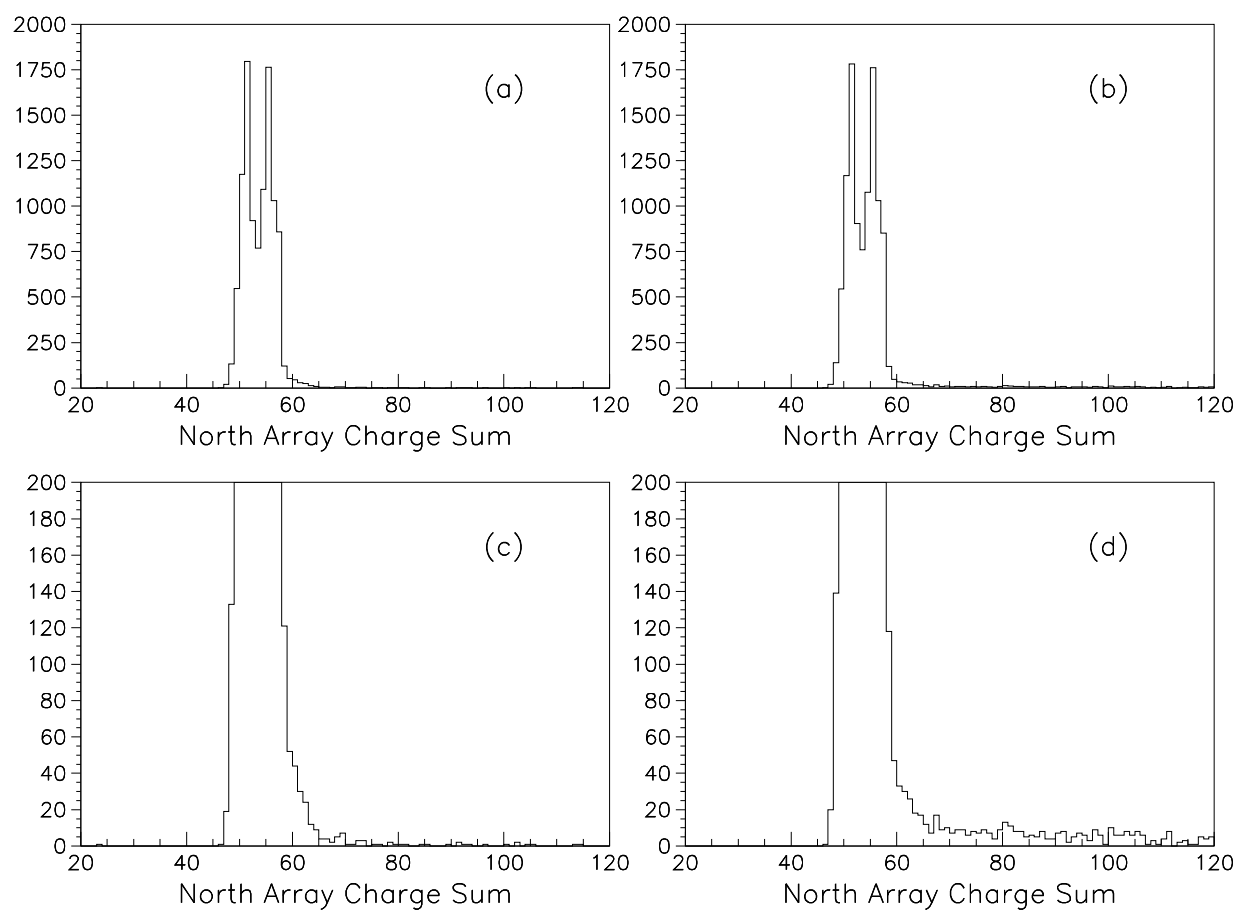

Figure 1: Comparison of the pedestal distribution of the analog charge sum from the LØ north array using (a) the standard cut on L $\emptyset$ pulse times and using (b) the no central tracking vertex found requirement for zero bias data taken at $7.5 \times 10^{30} \mathrm{~cm}^{-2} \mathrm{~s}^{-1}$ instantaneous luminosity. (c) and (d) are the same as (a) and (b), respectively, but with a magnified vertical scale.

no difference in shape. The plots with no central tracking vertex found show a long tail above the peak resulting from crossings in which an insufficient number of tracks was found in the central tracking region $(|\eta|<3.3)$. Note that the Level $\varnothing$ coverage extends well forward of central tracking so it is more sensitive to interactions with exclusively forward particles.

Figure 3 shows the charge distribution for the north (south) L $\emptyset$ array when an in-time hit is seen in the south (north) array. Figure 4 displays only the threshold region, with most of the events overflowing the plot. To eliminate events due to satellite-satellite collisions and beam halo, we require either a good FASTZ and in-time hits on both ends or a bad FASTZ and no 

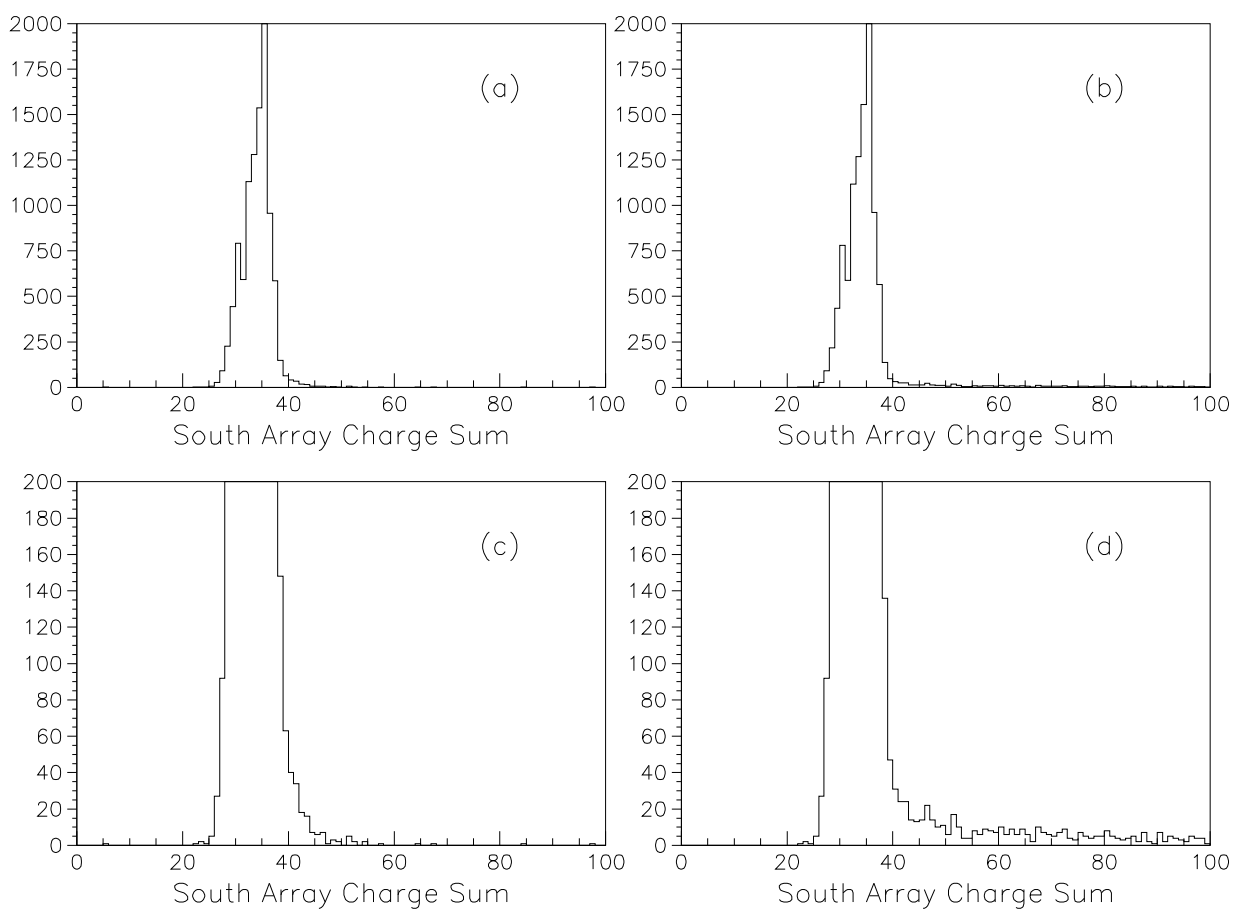

Figure 2: Comparison of the pedestal distribution of the analog charge sum from the $L \emptyset$ south array using $(a, c)$ the standard cut on $L \emptyset$ pulse times and using $(\mathrm{b}, \mathrm{d})$ the no central tracking vertex found requirement for zero bias data taken at $7.5 \times 10^{30} \mathrm{~cm}^{-2} \mathrm{~s}^{-1}$ instantaneous luminosity. 

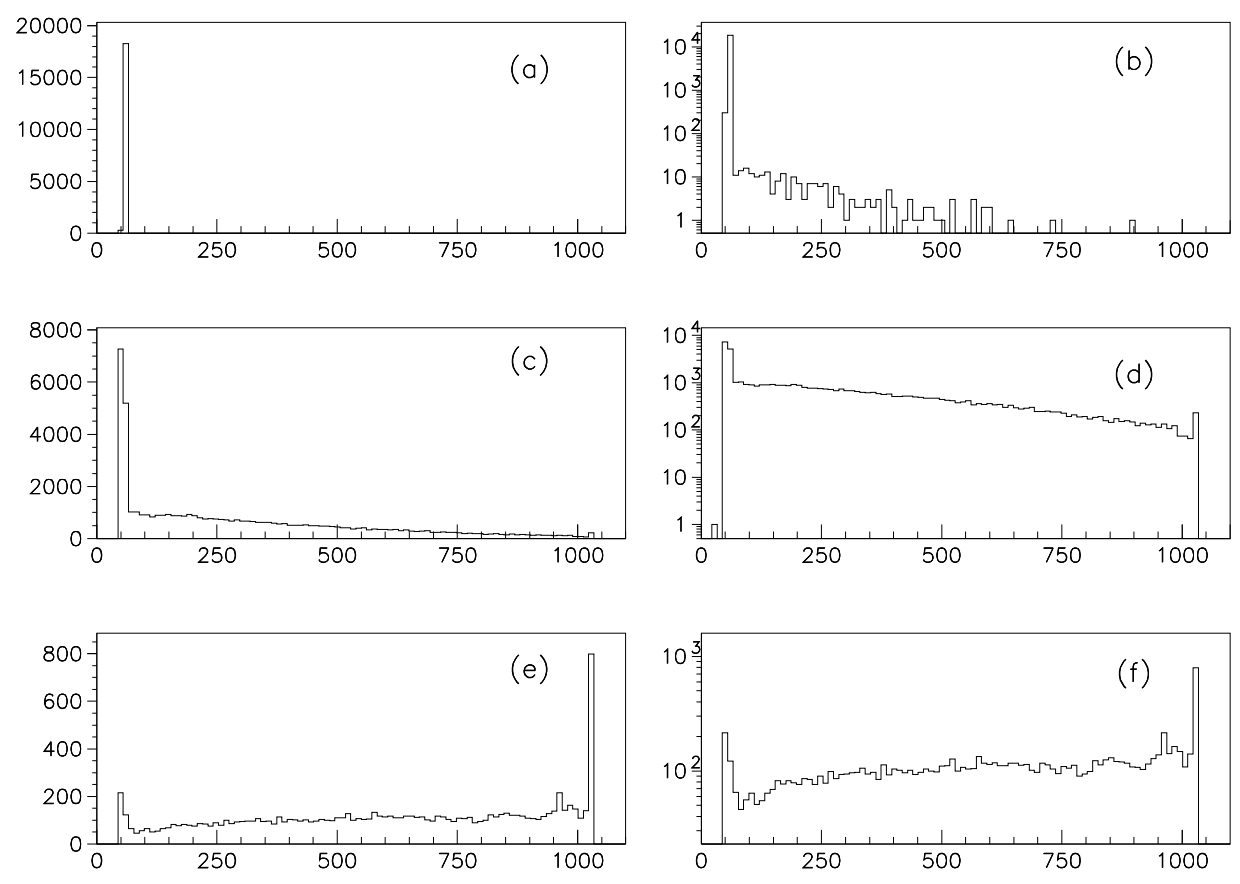

Figure 3: The full spectrum of the analog charge sum from the LØ north array in linear and $\log$ scale for zero bias samples taken at $(a, b) 0.07,(c, d)$ 7.5 , and $(\mathrm{e}, \mathrm{f}) 20.0 \times 10^{30} \mathrm{~cm}^{-2} \mathrm{~s}^{-1}$ instantaneous luminosities. Note that the highest bin with data contains all of the overflow charge sums.

hits within $100 \mathrm{~ns}$ of being in-time. This requirement also excludes events with $|z|>96 \mathrm{~cm}$; an inefficiency common to both the luminosity counting and physics triggers, thus having no effect on cross section measurements. Figure 4 shows a pedestal peak due to crossings where no particles hit the LØ array on top of a smooth charge distribution from events where both arrays are hit. The charge distributions for events with a good FASTZ are indicated by the hatched region, events with a good SLOWZ shown by the shaded region.

To estimate the LØ efficiency, we subtract the pedestal peak of Fig. 1(a) from Fig. 4(a), with a normalization determined using the central bins in the pedestal peak. The major uncertainty in this subtraction is the determination 

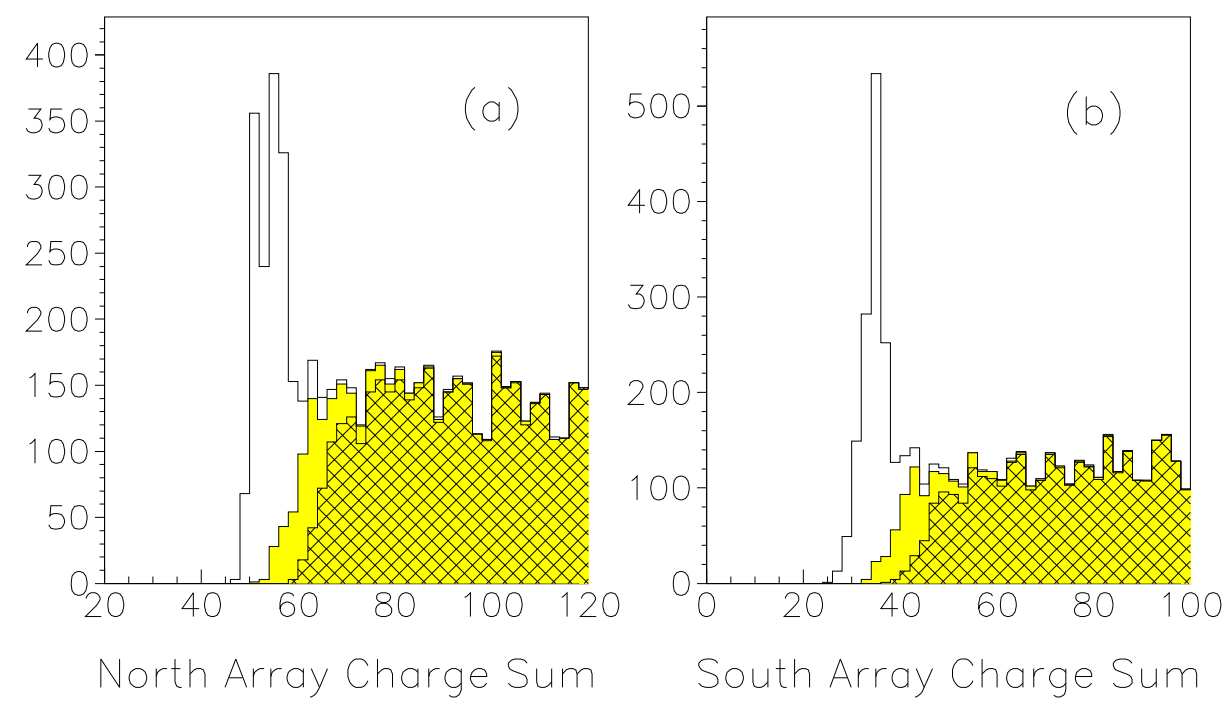

Figure 4: The analog charge sum from the L $\emptyset$ north and south arrays showing all events with hits on the opposite array indicating those events with a good FASTZ (hatched) and good SLOWZ (shaded) for a zero bias sample taken at $7.5 \times 10^{30} \mathrm{~cm}^{-2} \mathrm{~s}^{-1}$ instantaneous luminosity. 
of the number of events that have a particle hitting the L $\emptyset$ array with a charge in the normalization region. We assume the charge distribution for these events is $50 \%$ of the magnitude obtained by averaging the bins just above the pedestal peak bins and assign a $100 \%$ error to the magnitude obtained, i.e. $50 \% \pm 50 \%$. After subtracting the pedestal peak, the fraction of events that have a good FASTZ determines the efficiency for the north and south arrays. The final L $\emptyset$ efficiency is obtained by taking the product of the efficiencies of the north and south arrays. Poisson statistics are used to correct for the effects of multiple interactions as discussed in Sec 3. The results are shown in Fig. 5 and exhibit a residual luminosity dependence. The data are fitted to a line and the result is $\epsilon_{\mathrm{L} \emptyset}=0.897+0.00124 \mathcal{L}$ where $\mathcal{L}$ has units of $10^{30} \mathrm{~cm}^{-2} \mathrm{~s}^{-1}$. The fitted slope is used in the determination of the instantaneous luminosity. The complete 1994-1996 instantaneous luminosity distribution (Fig. 6) was used to compute the luminosity-weighted average $\epsilon_{\mathrm{L} \emptyset}=0.907 \pm 0.017$.

The $1994-1996 \epsilon_{\mathrm{L} \emptyset}$ is less than the $1992-1993$ value because a necessary electronics upgrade resulted in a slight reduction of the FASTZ signal amplitude relative to the discriminator threshold. Figure 7 shows the analog charge sum for the north array for the zero bias sample taken at $7.5 \times 10^{30} \mathrm{~cm}^{-2} \mathrm{~s}^{-1}$ instantaneous luminosity. The solid line is all of the data and the shaded region is the good FASTZ events. The dotted and dashed lines indicate the crossings that fired the SLOWZ with only one counter hit on the north side. Dotted failed FASTZ and the dashed passed good FASTZ. The large number of crossings in the dotted distribution indicate that many L $\varnothing$ counters hit with a single MIP do not have a large enough signal to pass the FASTZ discriminator threshold despite firing for SLOWZ. This was a smaller problem during the 1992-1993 Tevatron run before the electronics path upgrade. Figure 8 shows a comparison of data from the two running periods. The histograms show the distribution of the north array charge when at least one hit was seen in the south array. Again, the good FASTZ events are shaded. The events in which exactly one north array channel fired forming a valid SLOWZ event are shown in two distributions. These single channel events are divided into two groups, those passing FASTZ (dashed line) and those failing FASTZ (dotted line). Figure $8(\mathrm{~b})$ clearly shows a larger number of failures than Fig. 8(a) at the single counter level. Very few crossings with more than one counter hit failed FASTZ for either Tevatron run. 


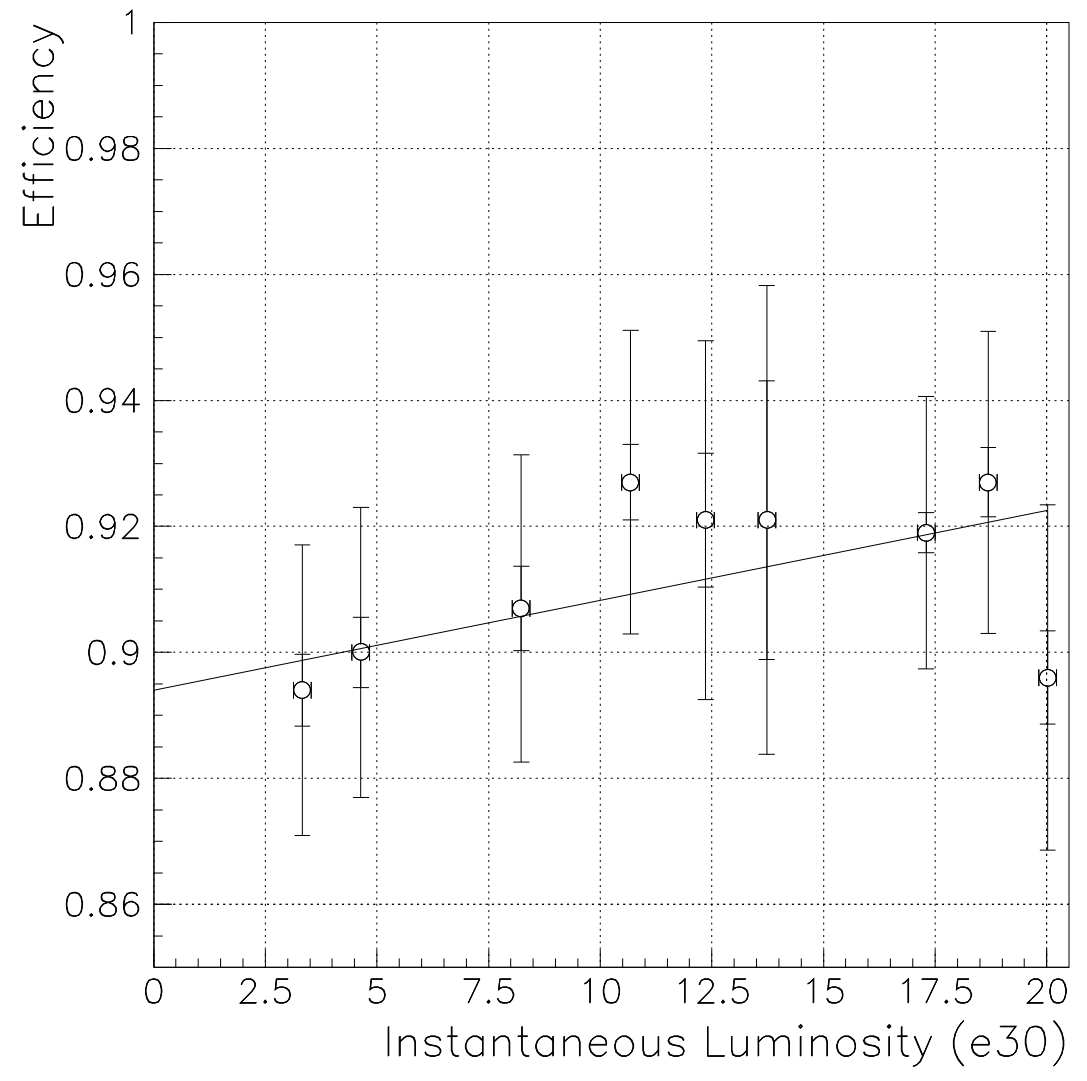

Figure 5: $\epsilon_{\mathrm{L} \emptyset}$ for each zero bias sample versus instantaneous luminosity. A line fit to the data shows a luminosity dependent bias where $\epsilon_{\mathrm{L} \emptyset}=0.897+$ $0.00124 \mathcal{L}$. 


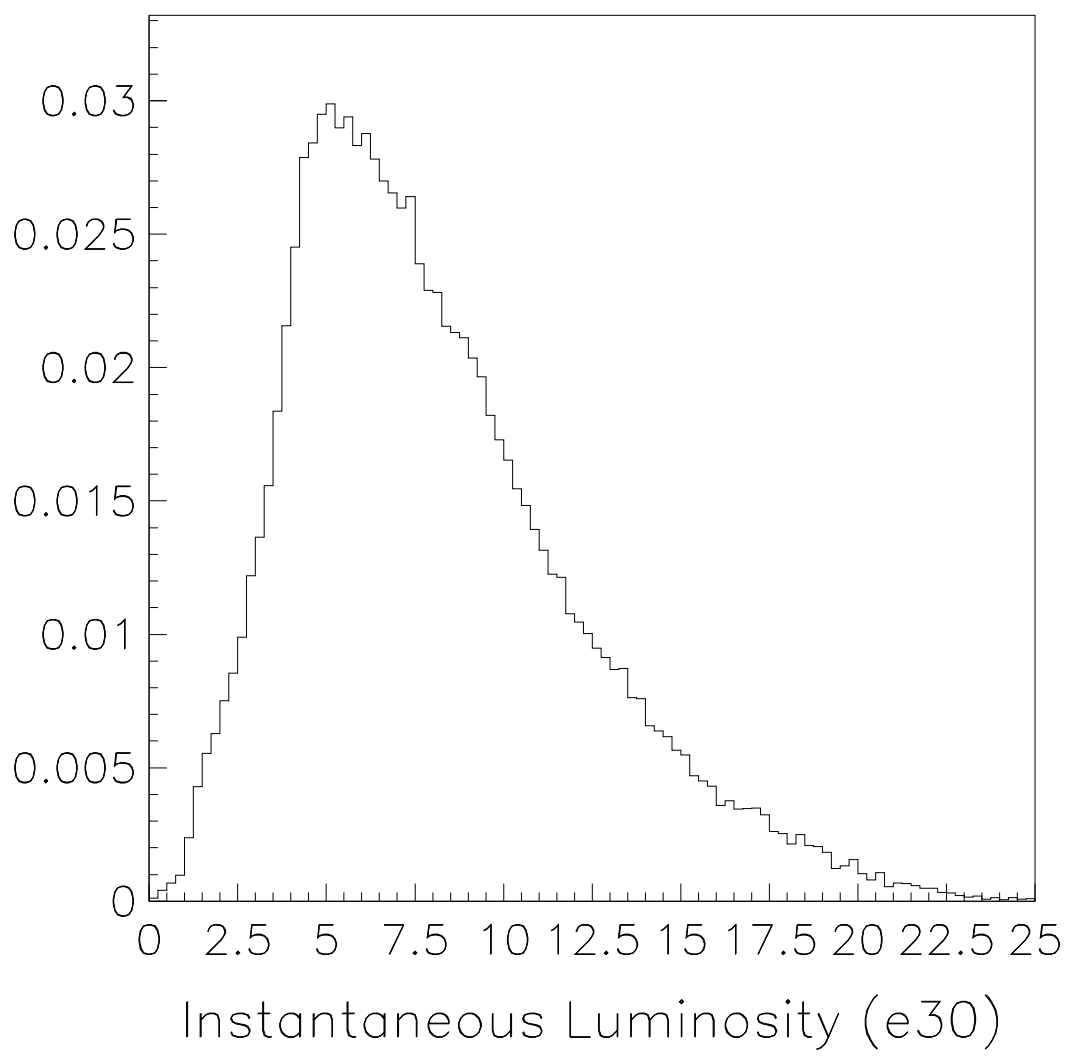

Figure 6: Distribution of the integrated luminosity per instantaneous luminosity bin for the 1994-1996 Tevatron run. 


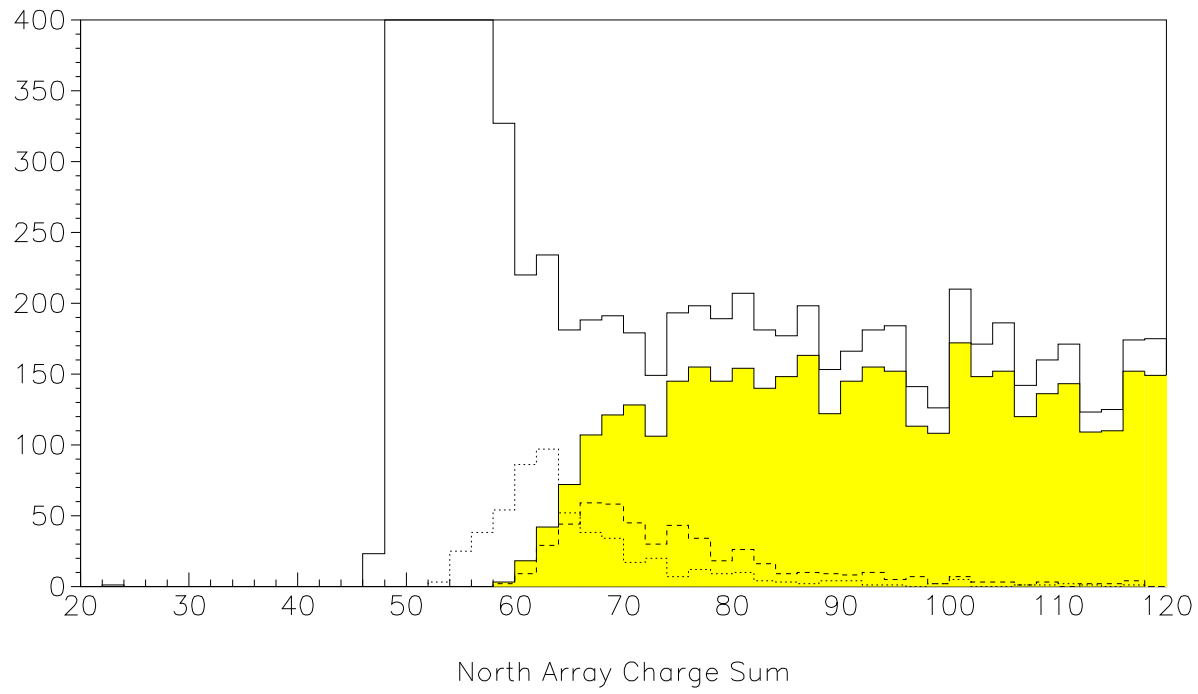

Figure 7: Comparison of the analog charge sum from the L $\emptyset$ north array for all events (solid line), good FASTZ events (shaded), single channel bad FASTZ events (dotted line), and single channel good FASTZ events (dashed line) in a zero bias sample taken at $7.5 \times 10^{30} \mathrm{~cm}^{-2} \mathrm{~s}^{-1}$ instantaneous luminosity. 


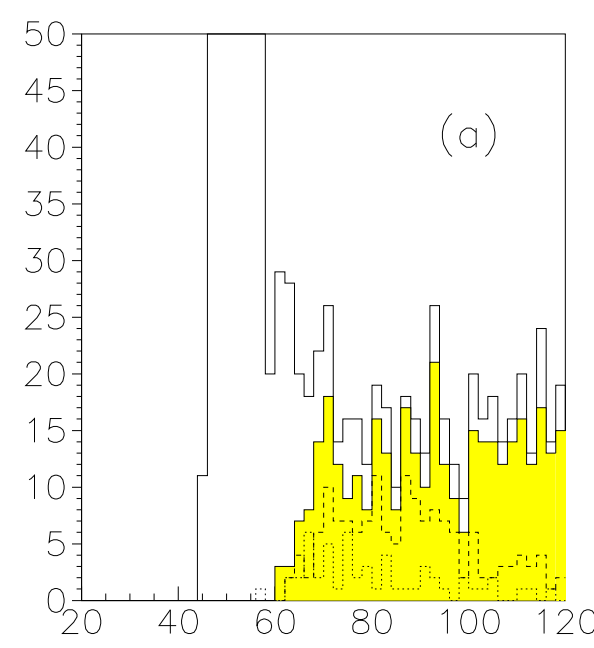

North Array Charge Sum

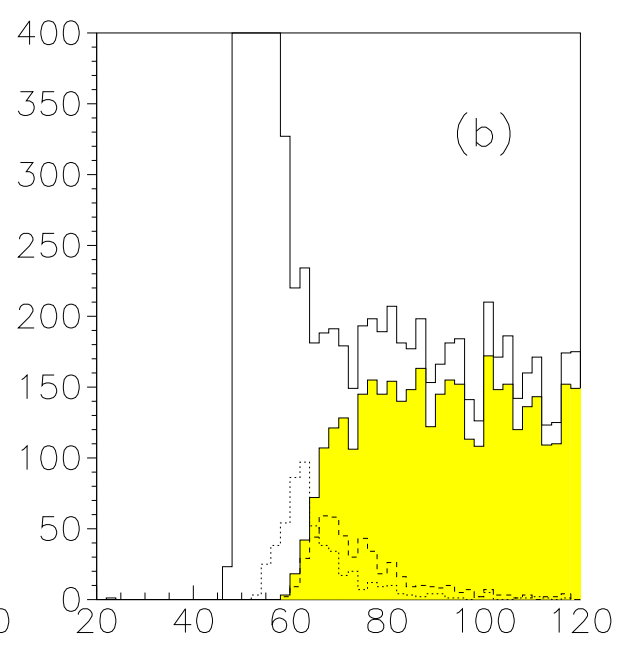

North Array Charge Sum

Figure 8: Comparison of the analog charge sum from the $L \emptyset$ north array for all events (solid line), good FASTZ events (shaded), single channel bad FASTZ events (dotted), and single channel good FASTZ events (dashed line) in zero bias samples taken during (a) the $1992-1993$ run at $5.0 \times 10^{30} \mathrm{~cm}^{-2} \mathrm{~s}^{-1}$ and during (b) the 1994-1996 run at $7.5 \times 10^{30} \mathrm{~cm}^{-2} \mathrm{~s}^{-1}$ instantaneous luminosity. 


\section{Correction for Multiple Single Diffractive Interactions}

The Level $\emptyset$ acceptance for single diffractive interactions is determined by the fraction of single diffractive interactions which are capable of having charged particles hit both arrays. The acceptance is low because diffraction products are infrequently emitted in the direction opposite to that of the initial beam particle. Thus, most single diffractive interactions would be one-sided, sending particles through one end array but not the other. In higher luminosity environments, it is possible to have multiple interactions with sufficient frequency that the rate of multiple single diffractive events is non-negligible. If the diffraction occurs in opposite directions for the two or more simultaneous interactions, then the acceptance becomes closer to the double diffractive acceptance value. Multiple one-sided single diffractive interactions in a crossing are not included in the overall acceptance calculation of Eqn. 1. A luminosity-dependent correction factor is required because these crossings can fire Level $\emptyset$ but are not considered in the acceptance calculation. Figure 9 shows the average correction factor versus instantaneous luminosity and Fig. 10 shows the average luminosity-weighted correction for all data recorded below the corresponding instantaneous luminosity.

\section{Correction for Beam Halo}

Beam halo is a second effect that becomes significant at the higher luminosities achieved in the 1994-1996 Tevatron run. Halo can veto a beam crossing thereby artificially lowering the luminosity. Although the accelerator ran at typically less than $1 \%$ halo (proton and anti-proton combined) during this period, the effect at the higher luminosities was significant. Since the LØ FASTZ was an integral part of physics triggers, no correction is needed for the linear portion of the halo term since a halo veto also vetoes the physics trigger. The halo factor remedies the error due to the effect of the multiple interaction correction portion of the halo term. The Level $\varnothing$ SLOWZ calculation was almost completely unaffected by halo and served as a reasonable baseline when determining the correction factor needed to remove the halo effect. Figure 11 shows the average halo correction factor versus instantaneous luminosity. Figure 12 shows the running halo correction for 


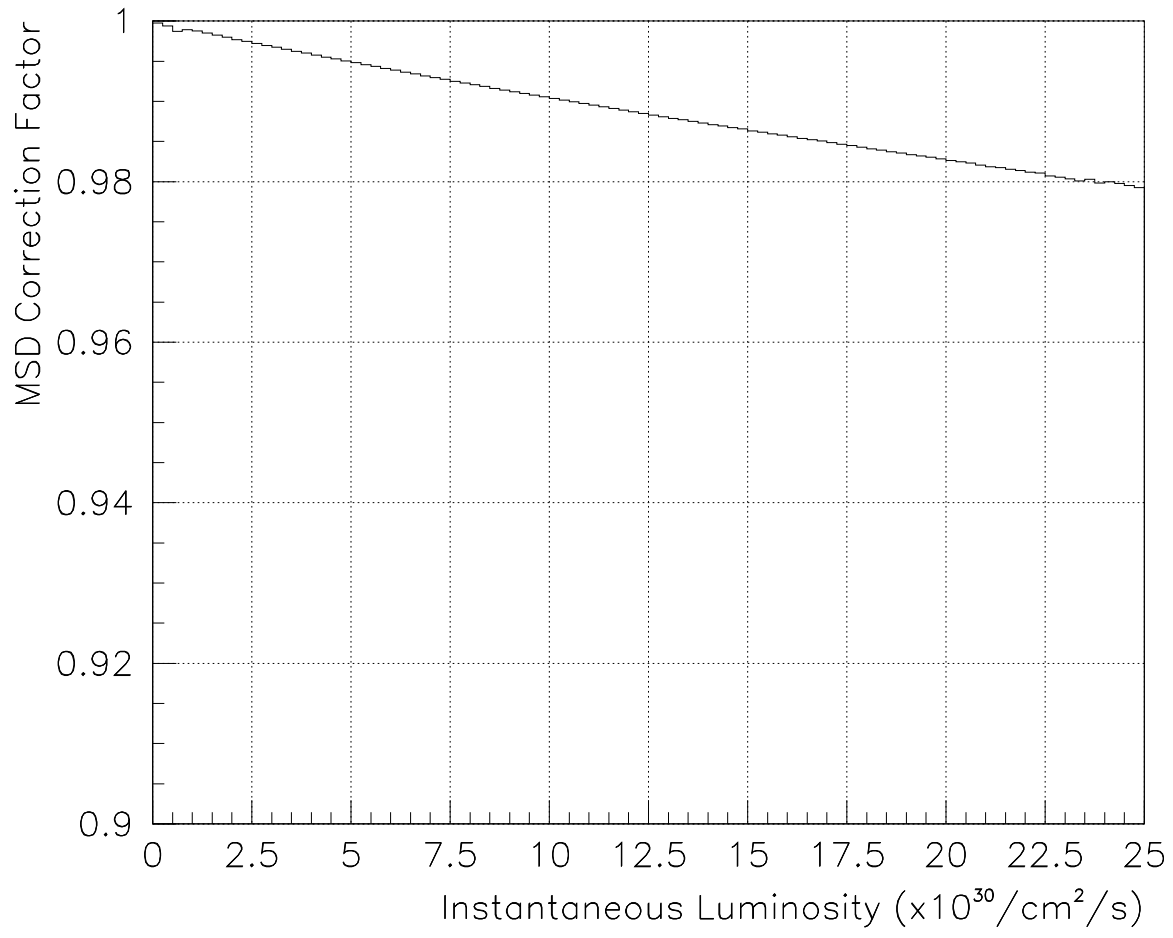

Figure 9: The average multiple single diffractive correction for the full range of instantaneous luminosities. 


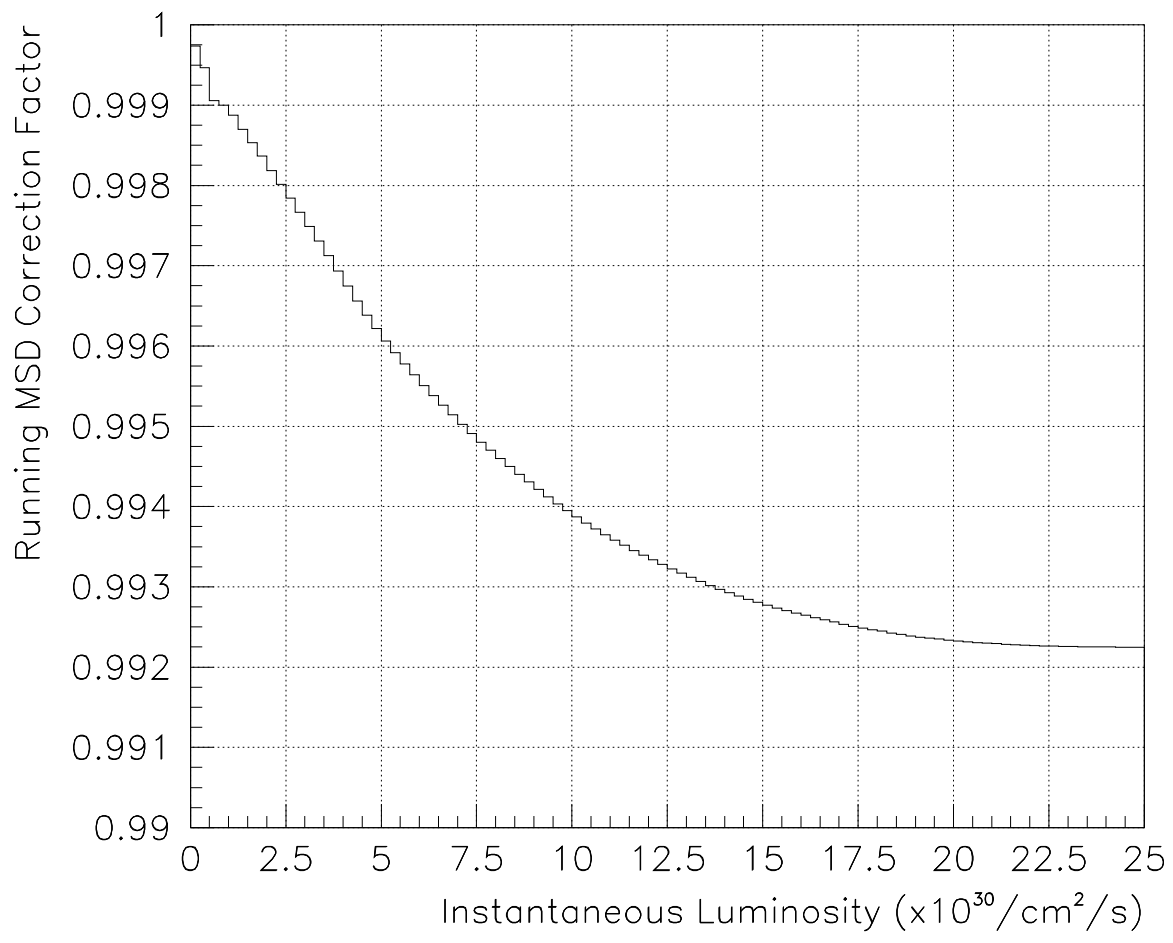

Figure 10: The running average multiple single diffractive correction at each instantaneous luminosity. 


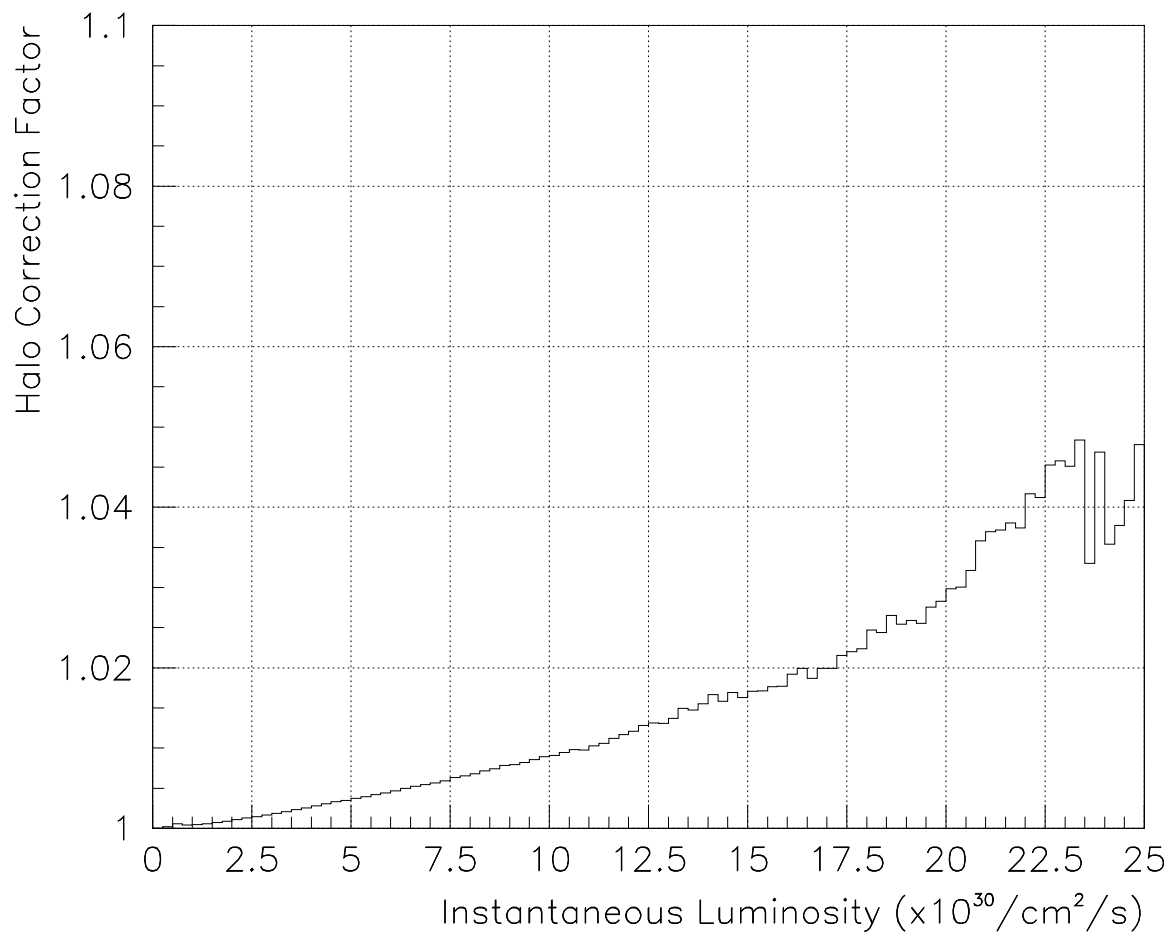

Figure 11: The beam halo correction for the full range of instantaneous luminosities.

the integrated luminosity calculation for all data up to a given instantaneous luminosity.

The halo and multiple single diffraction correction factors are roughly equal and opposite at most luminosities. Figure 13 shows the average combined correction factor for all data recorded below the corresponding instantaneous luminosity. At higher luminosities, the halo correction begins to dominate. A running correction is shown in Fig. 14 and the overall shift in integrated luminosity for the entire dataset is $\approx 0.03 \%$. Although this overall correction seems small, the correction for datasets obtained at predominately lower luminosities is larger. 


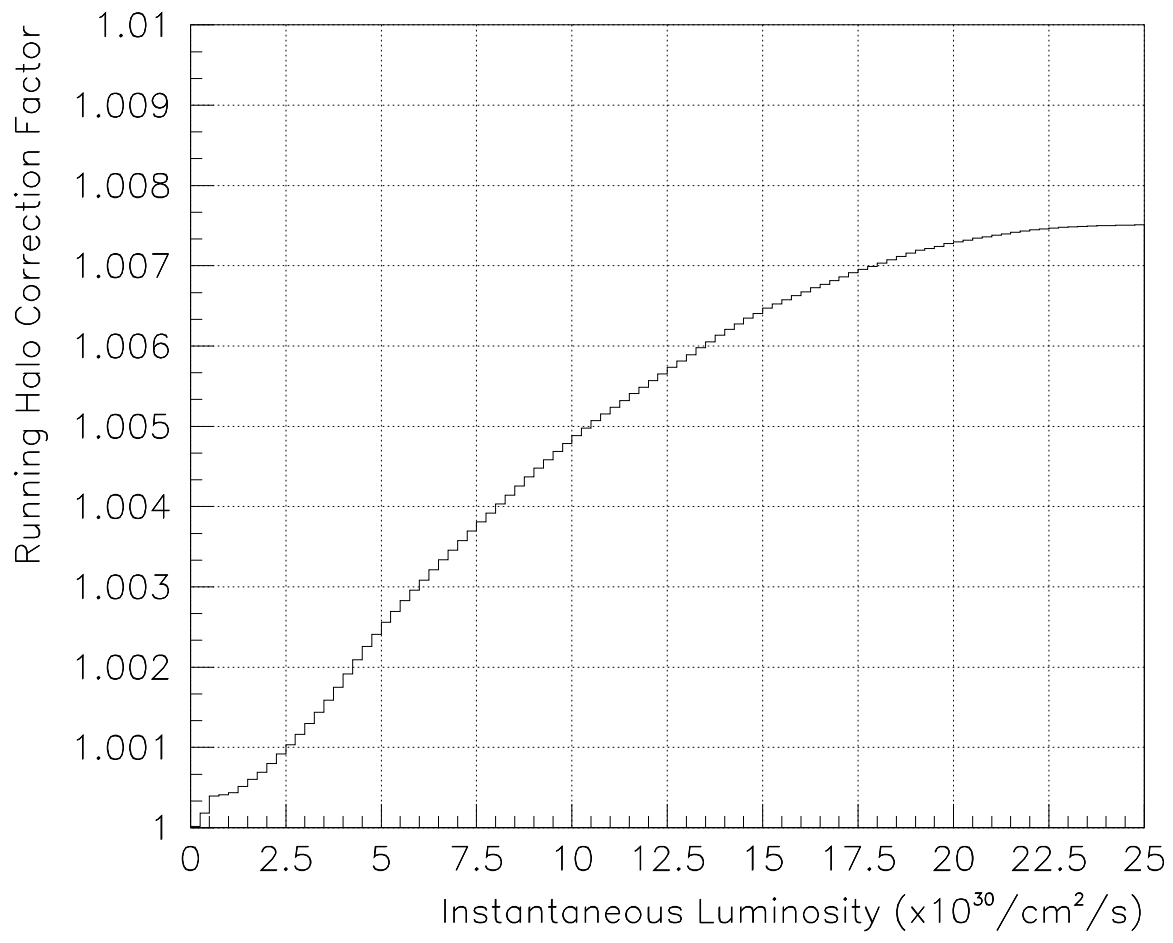

Figure 12: The running beam halo correction at each instantaneous luminosity. 


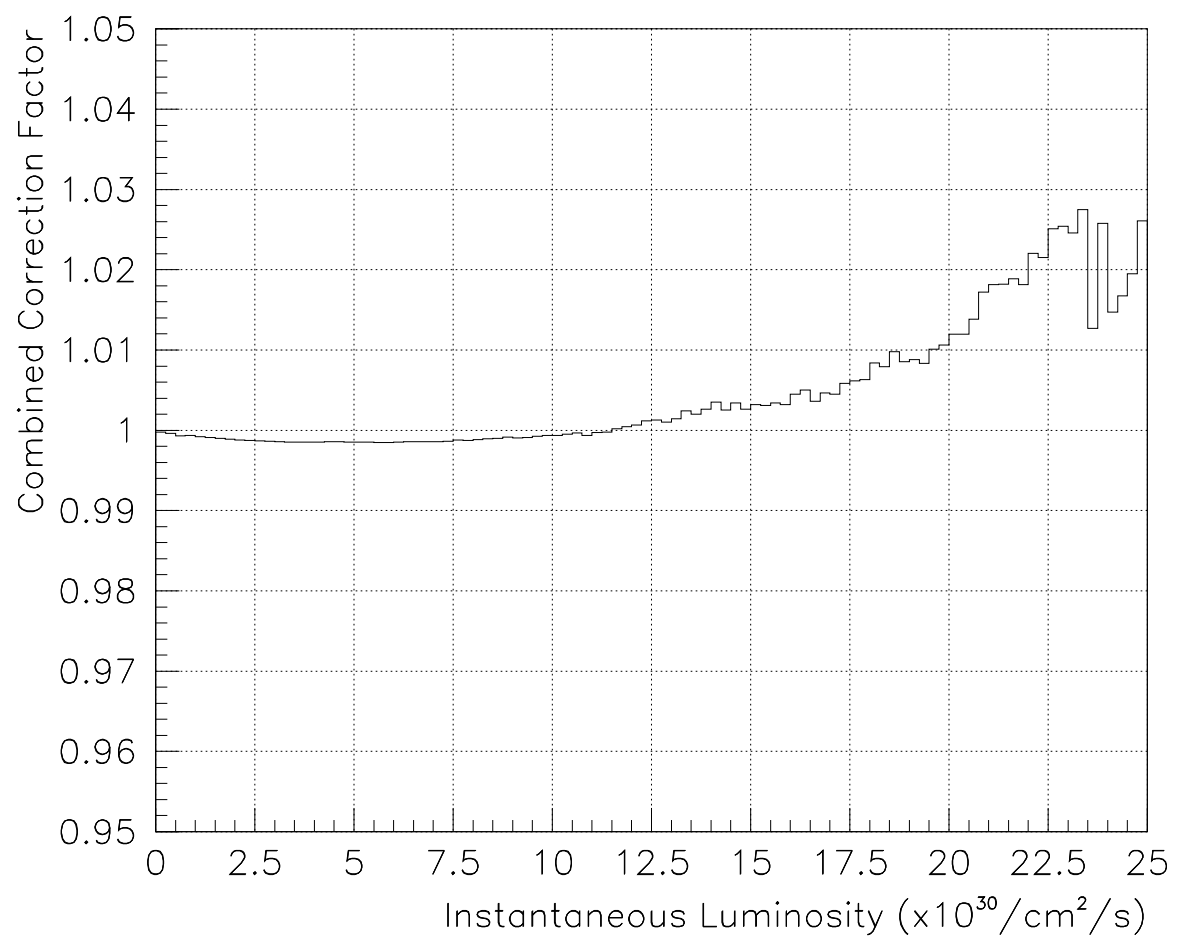

Figure 13: The combined halo and multiple single diffractive corrections for the full range of instantaneous luminosities. 


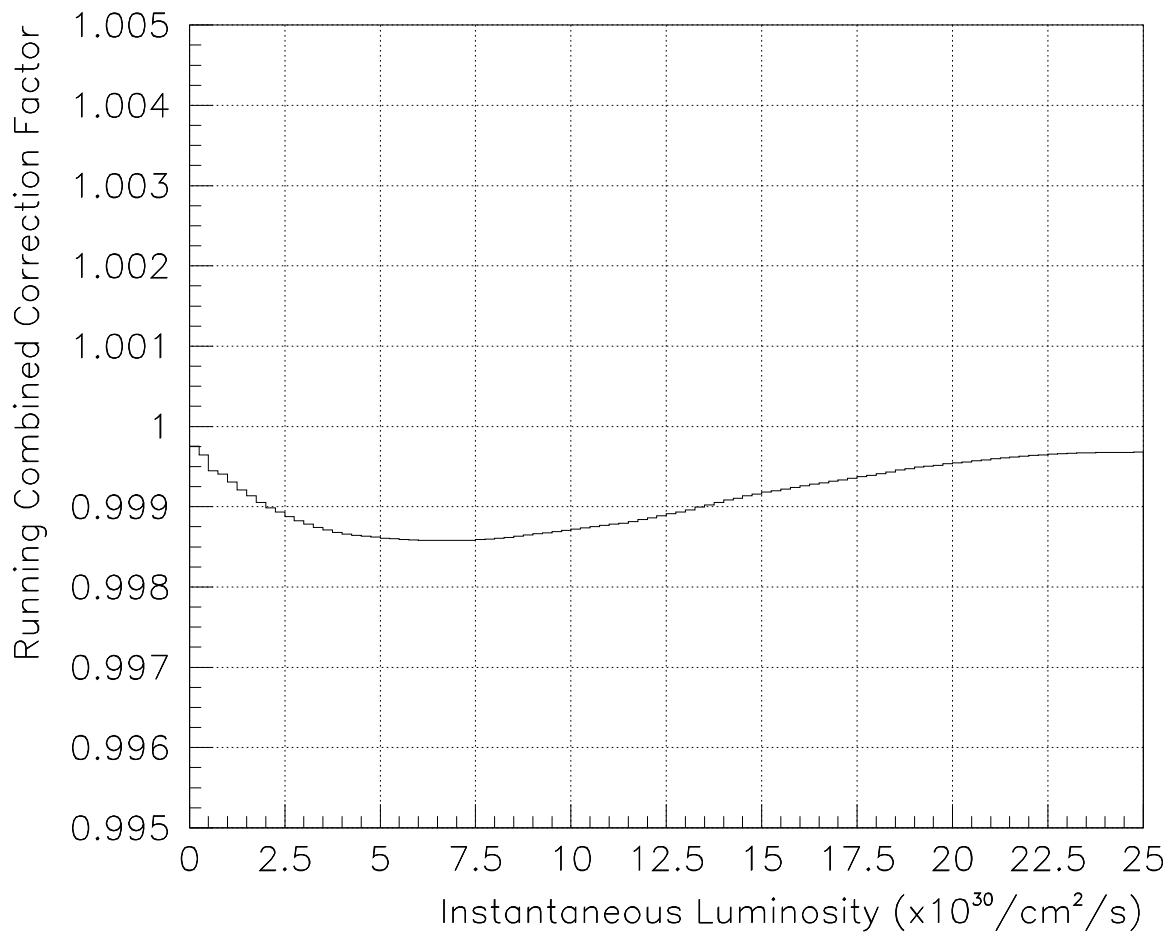

Figure 14: The running combined halo and multiple single diffractive corrections at each instantaneous luminosity. Note that the largest correction is for the full data set below $\approx 7.0 \times 10^{30} \mathrm{~cm}^{-2} \mathrm{~s}^{-1}$ instantaneous luminosity inclusive. 


\section{Determination of the Luminosity Monitor Constant}

The calculation of $\sigma_{\mathrm{L} \varnothing}$ requires the values in Appendix A.

$$
\sigma_{\mathrm{L} \emptyset}=\epsilon_{\mathrm{L} \emptyset} f_{\mathrm{halo}} f_{\mathrm{MSD}}\left(\epsilon_{\mathrm{SD}} \sigma_{\mathrm{SD}}+\epsilon_{\mathrm{DD}} \sigma_{\mathrm{DD}}+\epsilon_{\mathrm{HC}} \sigma_{\mathrm{HC}}\right)
$$

where $\epsilon_{\mathrm{L} \varnothing}, f_{\text {halo }}$, and $f_{\mathrm{MSD}}$ are functions of instantaneous luminosity. The results of the calculation expressed in Eqn. 7 are shown in Table 1 where the luminosity-weighted $\sigma_{\mathrm{L} \emptyset}$ and the full run's halo and MSD correction factors have been used.

\begin{tabular}{|l|c|c|}
\hline \hline & Mean & Error \\
\hline \hline$\left(\epsilon_{\mathrm{SD}} \sigma_{\mathrm{SD}}+\epsilon_{\mathrm{DD}} \sigma_{\mathrm{DD}}+\epsilon_{\mathrm{HC}} \sigma_{\mathrm{HC}}\right)$ & $49.11 \mathrm{mb}$ & $2.45 \mathrm{mb}$ \\
$\epsilon_{\mathrm{L} \varnothing}$ & 0.907 & $\mathbf{0 . 0 1 7}$ \\
\hline$f_{\text {halo }} \times f_{\mathrm{MSD}}$ & $\mathbf{0 . 9 9 9 7 5}$ & $\mathbf{0 . 0 0 2 0}$ \\
\hline$\sigma_{\mathrm{L} \varnothing}$ & $44.53 \mathrm{mb}$ & $2.37 \mathrm{mb}$ \\
\hline \hline
\end{tabular}

Table 1: Results of the calculation of the luminosity-weighted average $\sigma_{\mathrm{L} \varnothing}$ for the 1994-1996 Tevatron run.

\section{Conclusions}

For the 1994-1996 Fermilab collider run, the observable cross section for the $\mathrm{L} \emptyset$ counters, $\sigma_{\mathrm{L} \emptyset}=44.53 \pm 2.37 \mathrm{mb}$ was calculated using the world average for the total, elastic, and single diffractive cross sections, the earlier LØ acceptances, and the more accurate determinations of the L $\emptyset$ trigger efficiency factor. The effective $\sigma_{\mathrm{L} \emptyset}$ has a small residual luminosity dependence. This effect is included in integrated luminosity calculations along with corrections for multiple single diffractive interactions and beam halo effects. The additional corrections combined are less than $1 \%$ for almost all instantaneous luminosities, the running combined correction is less than $0.2 \%$ for any instantaneous luminosity on average. The effect for an individual Tevatron store can be much larger. 
We thank the staffs at Fermilab and the collaborating institutions for their contributions to the success of this work, and acknowledge support from the Department of Energy and National Science Foundation (U.S.A.), Commissariat à L'Energie Atomique (France), Ministries for Atomic Energy and Science and Technology Policy (Russia), CNPq (Brazil), Departments of Atomic Energy and Science and Education (India), Colciencias (Colombia), CONACyT (Mexico), Ministry of Education and KOSEF (Korea), CONICET and UBACyT (Argentina), and the A.P. Sloan Foundation.

\section{References}

[1] S. Abachi, et al., "The DØ Detector", Nucl. Inst. and Meth., A338 (issue 2/3) 185-253 (1994).

[2] J. Bantly, et al., "Improvement to the DØ Luminosity Monitor Constant", Fermilab Tech. Memo. TM-1930 (1996).

[3] J. Bantly, K. Epstein, J. Fowler, G.S. Gao, R. Hlustick, R.E. Lanou, F. Nang, R. Partridge, L. Wang, and H. Xu, IEEE Trans. on Nucl. Sci. 41, 1274 (1994).

[4] N. Amos, et al., "Change to the DØ Luminosity Monitor Constant", Fermilab Tech. Memo. TM-1911 (1995).

[5] M. Tartaglia, "Run 1B Tevatron Lattice Parameter Measurements with Separated Beams at DØ", Fermilab Tech. Memo. TM-1932 (in preparation). 


\section{A L $\varnothing$ Acceptances and World Average Cross Sections at $1.8 \mathrm{TeV}$}

The following tables contain results previously used in Ref. [2].

\begin{tabular}{|l|c|c|c|}
\hline \hline Inelastic Process & Mean (\%) & Stat Error (\%) & Sys Error (\%) \\
\hline SD Acceptance $\left(\epsilon_{\mathrm{SD}}\right)$ & 15.1 & 0.8 & 5.4 \\
DD Acceptance $\left(\epsilon_{\mathrm{DD}}\right)$ & 71.6 & 1.1 & 3.1 \\
HC Acceptance $\left(\epsilon_{\mathrm{HC}}\right)$ & 97.1 & 0.5 & 1.9 \\
\hline
\end{tabular}

Table 2: Results of the Monte Carlo L $\emptyset$ acceptances.

\begin{tabular}{|l|c|c|}
\hline \hline & Mean & Error \\
\hline \hline Inelastic Cross Section $\left(\sigma_{\text {Inelastic }}\right)$ & $58.94 \mathrm{mb}$ & $2.19 \mathrm{mb}$ \\
SD Cross Section $\left(\sigma_{\mathrm{SD}}\right)$ & $9.54 \mathrm{mb}$ & $0.43 \mathrm{mb}$ \\
DD Cross Section $\left(\sigma_{\mathrm{DD}}\right)$ & $1.15 \mathrm{mb}$ & $0.17 \mathrm{mb}$ \\
HC Cross Section $\left(\sigma_{\mathrm{HC}}\right)$ & $48.25 \mathrm{mb}$ & $2.23 \mathrm{mb}$ \\
\hline
\end{tabular}

Table 3: Cross Sections used in the $\sigma_{\mathrm{L} \emptyset}$ calculation.

\begin{tabular}{|l|c|c|}
\hline \hline & Mean & Error \\
\hline \hline$\epsilon_{\mathrm{SD}} \sigma_{\mathrm{SD}}$ & $1.44 \mathrm{mb}$ & $0.53 \mathrm{mb}$ \\
$\epsilon_{\mathrm{DD}} \sigma_{\mathrm{DD}}$ & $0.82 \mathrm{mb}$ & $0.094 \mathrm{mb}$ \\
$\epsilon_{\mathrm{HC}} \sigma_{\mathrm{HC}}$ & $46.85 \mathrm{mb}$ & $2.39 \mathrm{mb}$ \\
\hline$\left(\epsilon_{\mathrm{SD}} \sigma_{\mathrm{SD}}+\epsilon_{\mathrm{DD}} \sigma_{\mathrm{DD}}+\epsilon_{\mathrm{HC}} \sigma_{\mathrm{HC}}\right)$ & $49.11 \mathrm{mb}$ & $2.45 \mathrm{mb}$ \\
$\epsilon_{\mathrm{L} \emptyset}$ & 0.95 & 0.02 \\
\hline$\sigma_{\mathrm{L} \emptyset}$ & $46.7 \mathrm{mb}$ & $2.5 \mathrm{mb}$ \\
\hline \hline
\end{tabular}

Table 4: Results of the calculation of $\sigma_{\mathrm{L} \emptyset}$ for the 1992-1993 Tevatron run. 